Short Note

\title{
Application of random eigenvalue analysis to assess bridge flutter probability
}

\author{
Thomas Canor ${ }^{\mathrm{a}, \mathrm{b}}$, Luca Caracoglia ${ }^{\mathrm{c}}$, Vincent Denoël ${ }^{\mathrm{b}, *}$ \\ ${ }^{a}$ F.R.S.-FNRS, National Fund for Scientific Research, 1000 Bruxelles, Belgium \\ ${ }^{\mathrm{b}}$ University of Liege, Department of Architecture, Geology, Environment and Constructions, Structural Engineering Division, Chemin des Chevreuils, 1, B52/3, \\ 4000 Liege, Belgium \\ c Department of Civil and Environmental Engineering, 400 Snell Engineering Center, Northeastern University, 360 Huntington Avenue, Boston, MA 02115, USA
}

\section{A R T I C L E I N F O}

\section{Article history:}

Received 1 October 2014

Received in revised form

30 January 2015

Accepted 7 February 2015

\section{Keywords:}

Long-span bridges

Aeroelastic instability

Random perturbation

Collocation methods

Galerkin approach

Flutter probability

\begin{abstract}
A B S T R A C T
This study examines the well-known issue of evaluating the flutter probability for a long-span bridge, necessary for assessing structural integrity and reliability. The motivation stems from the need for critically reviewing and investigating a number of existing numerical approaches (e.g., random perturbation analysis, collocation methods, Galerkin approach) for the modeling and the solution of stochastic dynamic problems, by adapting them to the specific problem. The study proposes a generalized formulation for stochastic bridge flutter in terms of random eigenvalue analysis. A $1200 \mathrm{~m}$ suspension bridge model is used in the numerical simulations to compare the various methods and to provide indications on advantages and limitations of each method. Moreover, the link between the proposed formulation and existing studies on the propagation of uncertainty in aeroelastic systems, for example associated with measurement errors in wind tunnel, is examined.
\end{abstract}

(c) 2015 Elsevier Ltd. All rights reserved.

\section{Introduction and problem statement}

The study of bridge flutter has attracted the attention of researchers and designers for many decades due to the susceptibility to flutterinduced catastrophic structural failure. In line with the technological developments in long-span bridge design and construction, more and more sophisticated models for predicting the onset of flutter are necessary. Among these models, the use of probability-based analysis to study flutter occurrence has been often considered.

For example, probabilistic methods have been used to investigate bridge structural reliability, associated with the ultimate collapse limit state induced by flutter (e.g., Cheng et al., 2005; Ge et al., 2000; Pourzeynali and Datta, 2002; Dragomirescu et al., 2003). On the other hand, stochastic calculus has been employed to examine the effects of atmospheric turbulence either on flutter onset (e.g., Bucher and Lin, 1988; Sarkar and Tsiatas, 2009; Bartoli et al., 1997; Cai and Albrecht, 2000; Sepe and Vasta, 2014) and beyond, i.e., in a post-critical state (Hračov et al., 2005; Náprstek and Pospíšil, 2004; Pospíšil et al., 2006).

In recent years, capitalizing from the pioneering results on turbulence effects on flutter (e.g., Bucher and Lin, 1988), stochastic

\footnotetext{
* Corresponding author.

E-mail addresses: t.canor@ulg.ac.be (T. Canor), lucac@coe.neu.edu (L. Caracoglia),v.denoel@ulg.ac.be (V. Denoël).

bridge flutter analysis has been used to examine the effect of various sources of uncertainty (modeling and measurement error propagation), originating for example from wind tunnel tests on a section model of a bridge deck. These sources can include errors in the estimation of aeroelastic loads (Caracoglia, 2011). Incidentally, the issue of error analysis and propagation had been overlooked and seldom investigated, except for a limited number of cases (e.g., Caracoglia, 2011). Nevertheless, recent studies (Sarkar et al., 2009; Kwon, 2010; Mannini and Bartoli, 2012; Argentini et al., 2014) have reaffirmed the need for ensuring that uncertainty evaluation, while conducting an experiment to determine aeroelastic loads (e.g., flutter derivatives), is carefully considered and included in flutter predictions.

Building on previous investigations and literature results, this study aims at critically reviewing and examining a number of analysis and solution methods, applied to the prediction of long-span bridge flutter probability. The three main objectives of the work are: (i) to propose the use of a randomly parametrized matrix and system for the study of stochastic bridge flutter, in which random parameters are used to describe aeroelastic force measurement errors; (ii) to estimate flutter probability by using a possibly more general analysis framework, i.e., in the context of random eigenvalue analysis, especially when the number of random parameters is large; (iii) to investigate a series of numerical analysis tools and solution methods for stochastic bridge flutter simulation, as alternatives to MonteCarlo sampling; these methods include: perturbation approach, stochastic collocation 
(Le Maître and Knio, 2010) and Galerkin approach (Ghanem and Spanos, 1991; Ghanem and Ghosh, 2007).

A $1200 \mathrm{~m}$ long-span bridge, equipped with truss-type deck girder, is utilized as application example. Both single-mode torsional flutter and coupled-mode flutter are examined. Even though the use of sampling methods is often recommended (e.g., Xu, 2013) for systems with several random variables, other methods such as the stochastic collocation can still provide sufficiently adequate results.

\section{Reduced-order model for dynamic analysis of bridges}

The dynamic response of a long-span bridge was derived from the multi-mode formulation in the frequency domain (Jones and Scanlan, 2001). Wind-induced forces can be represented as a superposition of aeroelastic (motion-induced) and aerodynamic (turbulence-induced) loads. The equations of motion of a long-span bridge subject to wind loading are given by

$m \partial_{t}^{2} h+c_{y} \partial_{t} h+k_{h} h=L_{a e}+L_{b}$

$I_{\alpha} \partial_{t}^{2} \alpha+c_{\alpha} \partial_{t} \alpha+k_{\alpha} \alpha=M_{a e}+M_{b}$

with $h(t, x)$ and $\alpha(t, x)$ the vertical displacement and the rotation angle of the bridge deck along the span at coordinate $x$, respectively. The coefficients $c_{y}, c_{\alpha}, k_{y}$ and $k_{\alpha}$ refer to the viscous damping and stiffness in the corresponding directions of vibration. The horizontal displacement (bridge sway) is not considered in the present work. The vibrations of the structure are assumed to be small. The wind loading is split into two contributions: (i) the aeroelastic lift force $\left(L_{a e}\right)$ and torsional moment $\left(M_{a e}\right)$ and (ii) the buffeting lift force $\left(L_{b}\right)$ and torsional moment $\left(M_{b}\right)$. These two sources are supposed to be decoupled and not to interact with each other (Jones and Scanlan, 2001). In the multi-mode formulation, the displacement components are represented via a modal expansion derived from the unloaded structure, such that

$h(t, x)=\sum_{j} B \eta_{j}(t) h_{j}(x)$,
$\alpha(t, x)=\sum_{j} \eta_{j}(t) \alpha_{j}(x)$

with $h_{j}$ and $\alpha_{j}$ the dimensionless vertical and torsional components of the $j$ th mode and $B$ the deck width.

The aeroelastic loads per unit length can be interpreted as parametric excitations depending on structural vibration via flutter derivatives, such that

$L_{\mathrm{ae}}=\mathrm{qB}\left[\mathrm{KH}_{1}^{*} \frac{1}{U} \partial_{t} h+\mathrm{KH}_{2}^{*} \frac{B}{U} \partial_{t} \alpha+K^{2} H_{3}^{*} \alpha+K^{2} H_{4}^{*} \frac{h}{B}\right]$,

$M_{a e}=q B^{2}\left[K A_{1}^{*} \frac{1}{U} \partial_{t} h+K A_{2}^{*} \frac{B}{U} \partial_{t} \alpha+K^{2} A_{3}^{*} \alpha+K^{2} A_{4}^{*} \frac{h}{B}\right]$

with $q=\frac{1}{2} \rho U^{2}, \rho$ the air density, $U$ the mean wind velocity, $K=\omega B / U$ the reduced frequency and $\omega$ the pulsation. The coefficients $H_{i}^{*}(K)$ and $A_{i}^{*}(K)(i=1, \ldots 4)$, designated as flutter derivatives, are commonly identified in wind tunnel tests as functions of $K$. On the other hand, turbulence-induced forces are direct loads, usually modeled by a set of random processes, coherent in time and space. The buffeting forces are intrinsically random, while the aeroelastic forces are usually considered as deterministic. However, uncertainties in the flutter derivative measurements change this perspective to better interpret them as random processes in the frequency domain (e.g., Seo and Caracoglia, 2011, 2012).

The aeroelastic forces, simultaneously defined in time and frequency domains, may be converted by inverse Fourier transform into indicial functions, expressed as a function of dimensionless time $s=t U / B$ (Scanlan et al., 1974). An indicial function (IF) is a dimensionless function modeling the unsteady evolution of the aeroelastic loads,

$\Phi_{P p}(s)=1-\sum_{i=1} a_{i, P p} \exp \left(-b_{i, P p} s\right)$

with $P=\{L, M\}$ and $p=\{h, \alpha\}$. The parameters $a_{i, P p}$ and $b_{i, P p}$ result from a nonlinear fitting of the coefficients, for example as explained in Caracoglia and Jones (2003). The summation in (5) may contain from 1 to 4 terms depending on the complexity of the actual indicial function to be fitted. The aeroelastic loading may be simplified by introducing new variables $\{\mu, \nu\}$, known as the aeroelastic states, in order to describe the time evolution of the unsteady loading

$\dot{\mu}_{i, P h, j}=a_{i, P h} b_{i, P h} \dot{\eta}_{j}-b_{i, P h} \mu_{i, P h, j}$

$\dot{\nu}_{i, P \alpha, j}=a_{i, P \alpha} b_{i, P \alpha} \eta_{j}-b_{i, P \alpha} \nu_{i, P \alpha, j}$

with $P=\{M, L\}$. The dot symbol in the equations above designates the derivative with respect to $s$. Additional details are provided in Caracoglia (2011, 2013).

Therefore the final form of the equations of motion is

$$
\begin{aligned}
\frac{1}{Q_{j}} \ddot{\eta}_{j}= & -\frac{1}{Q_{j}}\left(2 \xi_{j} K_{j} \dot{\eta}_{j}+K_{j}^{2} \eta_{j}\right) \\
& +\sum_{k=1}^{M} \hat{C}_{L} G_{h_{j} h_{k}}\left[\Phi_{L h}(0) \dot{\eta}_{k}+\sum_{i=1}^{m_{L h}} \mu_{i, L h, k}\right] \\
& +\sum_{k=1}^{M} \hat{C}_{L} G_{h_{j} \alpha_{k}}\left[\Phi_{L \alpha}(0) \eta_{k}+\sum_{i=1}^{m_{L \alpha}} \nu_{i, L \alpha, k}\right] \\
& +\sum_{k=1}^{M} \hat{C}_{M} G_{\alpha_{j} h_{k}}\left[\Phi_{M h}(0) \dot{\eta}_{k}+\sum_{i=1}^{m_{M h}} \mu_{i, M h, k}\right] \\
& +\sum_{k=1}^{M} \hat{C}_{M} G_{\alpha_{j} \alpha_{k}}\left[\Phi_{M \alpha}(0) \eta_{k}+\sum_{i=1}^{m_{M \alpha}} \nu_{i, M \alpha, k}\right]
\end{aligned}
$$

with $G_{\alpha_{j} h_{k}}=(1 / l) \int_{0}^{l} \alpha_{j}(x) h_{k}(x) \mathrm{d} x, Q_{j}=\rho B^{4} l / 2 I_{j}$ with $I_{j}$ the generalized modal inertia and the reduced pulsation is $K_{j}=\omega_{j}(B / U)$. The modal coupling is estimated through the variable $G_{\alpha_{j} h_{k}}$ and $G_{\alpha_{j} \alpha_{k}}$ for different combinations of $j \neq k$. We also introduced the coefficients $\hat{C}_{L}=-\partial_{\alpha} C_{L}$ and $\hat{C}_{M}=\partial_{\alpha} C_{M}$.

Eqs. (6)-(8) can be recast into a state formalism with $\mathbf{X}(s)=$ $\left[\eta_{i}, \dot{\eta}_{i}, \mu_{i, P h, j}, \nu_{i, P \alpha, j}\right]$ and $\mathbf{F}_{b}(s)$ a vector of the turbulence-induced loads. The dynamical system can be rewritten, after simplification, as

$\dot{\mathbf{X}}=\mathbf{A X}+\mathbf{F}_{b}(s)$.

Matrix $\mathbf{A}$ is a time-invariant matrix gathering structural and aeroelastic parameters. An explicit expression of $\mathbf{A}$ is given in Caracoglia (2013). From (9), it is obvious that bounded processes, originating from buffeting loads in the exogenous term $\mathbf{F}_{b}$, are not sources of instability. In order for the contribution of the buffeting force to be relevant, a parametric perturbation to the mean wind speed would be required. This can be accomplished for instance by replacing the quantity $U$ with the quantity $U[1+w(t)]$ with $w(t)$ a random noise (Bucher and Lin, 1988; Sarkar and Tsiatas, 2009). Nevertheless, the use of such a parametric disturbance has been noted as not being fully representative of the physical phenomenon (Caracoglia, 2011) and that the effect of turbulence on flutter should be considered either through a loss of spanwise correlation of the aeroelastic loading (Scanlan, 1997) or by propagating through the system a parametric-type disturbance in the spanwise correlation of $\mathbf{F}_{b}$ (Caracoglia and Jones, 2003). Another approach (Caracoglia, 2013), which is compatible with the interpretation of the role of aeroelastic forces versus buffeting forces in the "classical" bridge aerodynamics formulation by Scanlan (1997), has also suggested that the role of buffeting force (and perturbation) is usually of secondary importance in comparison with the variability in the coefficients describing the aeroelastic loads. The formulation, used in this work, employs the model and the results in Caracoglia (2013) to justify the choice of neglecting the investigation of buffeting load 
effects.Therefore, for the purposes of this study, it is acceptable to assume that the two loading sources are decoupled and that instability is controlled by the eigenvalues of $\mathbf{A}$. As a result, a perturbation to the terms of this matrix will be applied in the next section to simulate uncertainty propagation on flutter, i.e. the influence of aeroelastic loading measurement errors.

\section{Stability of linear random systems}

\subsection{Basis of linear stability}

On a probability space $(\Theta, \mathfrak{F}, \mathbb{P})$, we consider a linear timeinvariant (LTI) system with random parameters, such as

\section{$\dot{\mathbf{X}}=\mathbf{B}(\theta) \mathbf{X}$}

with the random vector $\mathbf{X}(t, \theta): \mathbb{R}^{+} \times \Theta \mapsto \mathbb{R}^{n}$, the randomly parametrized matrix $\mathbf{B}(\theta): \Theta \mapsto \mathbb{R}^{n \times n}$ and $\mathbf{x}_{0}$ an initial condition (possibly random). We suggest to use the concept of randomly parametrized matrix, a distinction from the concept of random matrix, since the randomness in $\mathbf{B}(\theta)$ is inherited from randomness in the parameters of the system. The matrix B in Eq. (10) is the error-contaminated version of the error-free matrix $\mathbf{A}$, described in the previous section.

The stability of this linear system may be judged in the light of the eigenvalues $\lambda(\theta): \Theta \mapsto \mathbb{C}$ of $\mathbf{B}(\theta)$, i.e., the roots of the characteristic polynomial $\mathcal{P}(\lambda(\theta))$ defined as

$\mathcal{P}(\lambda(\theta))=\operatorname{det}(\mathbf{B}(\theta)-\lambda(\theta) \mathbf{I})$

with I the $n$-dimensional identity matrix. The $n$ eigenvalues satisfying this characteristic equation are gathered in $\boldsymbol{\Lambda}=$ $\left\{\lambda_{i} \mid i=1, \ldots n\right\} \in \mathbb{C}^{n}$. To each eigenvalue $\lambda_{i}(\theta)$ corresponds an eigenvector $\mathbf{Y}_{i}(\theta): \Theta \mapsto \mathbb{C}^{n}$, such that

$\mathbf{B}(\theta) \mathbf{Y}(\theta)=\lambda(\theta) \mathbf{Y}(\theta)$

with the normalization condition $\left\langle\mathbf{Y}_{i}, \mathbf{Y}_{j}\right\rangle=\delta_{i j}$. The randomness of $\mathbf{B}(\theta)$ induces randomness in the $n$ pair of random eigenvalues and eigenvectors $\left\{\lambda_{i}(\theta), \mathbf{Y}_{i}(\theta)\right\}$. Since $\mathbf{B}(\theta)$ is real-valued, the random characteristic polynomial $\mathcal{P}(\lambda(\theta))$ has only real random coefficients and the eigenvalues are real-valued or complex conjugate.

For a given realization, the system is stable provided all eigenvalues, associated with the generalized coordinates of the structural modes, have a negative real part. Thence, the stability of the system depends on the joint probability density function (PDF) of the vector $\mathfrak{R}(\boldsymbol{\Lambda}(\theta))$, designated by $p_{\Re(\boldsymbol{\Lambda})}(\theta): \Theta \mapsto \mathbb{R}_{0}^{+}$,

$\operatorname{Pr}($ stable $)=\operatorname{Pr}\left(\forall i, \lambda_{i}^{\Re}(\theta)<0\right)=\int_{\Theta} p_{\{\Re(\Lambda)<0\}}(\theta) \mathrm{d} \theta$,

where superscript $\mathfrak{R}$ denotes the real part of the argument. Computing this probability is the key goal of this work to assess the risk of bridge flutter instability. Three different methods will be considered in the sequel to compute this joint-PDF $p_{\Re(\boldsymbol{\Lambda})}(\theta)$ : (i) perturbation approach, (ii) collocation method, and (iii) Galerkin projection.

\subsection{Perturbation approach}

The perturbation approach consists in expanding any eigenvalue $\lambda_{i}$ in a Taylor series in terms of the random parameters around their mean value $\bar{\theta}_{i}$ up to a first or a second order, such as

$\lambda_{i}(\theta) \approx \bar{\lambda}_{i}+\sum_{j}^{N}\left(\theta_{j}-\bar{\theta}_{j}\right) \mathcal{J}_{j}^{\lambda_{i}}(\bar{\theta})+\frac{1}{2} \sum_{j}^{N} \sum_{k}^{N}\left(\theta_{j}-\bar{\theta}_{j}\right)\left(\theta_{k}-\bar{\theta}_{k}\right) \mathcal{H}_{k j}^{\lambda_{i}}(\bar{\theta})$.

The factors $\mathcal{J}_{j}^{\lambda_{i}}(\bar{\theta})=\left(\partial_{j} \lambda_{i}\right)_{\theta=\bar{\theta}}$ and $\mathcal{H}_{k j}^{\lambda_{i}}(\bar{\theta})=\left(\partial_{k j}^{2} \lambda_{i}\right)_{\theta=\bar{\theta}}$ denote the elements of the Jacobian and Hessian matrices, respectively. The pair $\bar{\lambda}_{i}$ and $\overline{\mathbf{Y}}_{i}$ results from the eigenproblem (12) by setting $\theta=\bar{\theta}$.
Adhikari and Friswell (2007) propose an iterative improvement of this method, by changing the point $\bar{\theta} \in \Theta$. However, the mean value $\bar{\theta}$ remains a convenient guess, especially for slightly dispersed random system, independently of the probability distribution of $\theta$. The Jacobian and Hessian matrices are typically estimated numerically. Closed forms can only be obtained for specific cases, e.g., when $\mathbf{B}(\theta)$ is a symmetric matrix. The Taylor expansion (14) is also simplified and both the Jacobian and Hessian matrices can be computed in closed form.

For small dispersion, the first-order Taylor expansion is a good approximation. Furthermore, if the random parameters are independent Gaussian variables, each variable $\theta_{i}$ can be expressed as $\theta_{i}=\bar{\theta}_{i}+\sigma_{\theta_{i}} \xi_{i}$ with $\Xi=\left\{\xi_{i} \mid i=1, \ldots n\right\}$ a set of independent standard Gaussian variables with $\sigma_{\theta_{i}}$ the standard deviation of $\theta_{i}$. The mean value of the real parts of $\lambda_{i}$ is $\boldsymbol{\mu}\left(\lambda_{i}^{\Re}\right) \approx \bar{\lambda}_{i}^{\Re}$, while the covariance matrix is approximated by

$\Sigma\left(\lambda_{i}^{\Re}, \lambda_{j}^{\Re}\right) \approx \sum_{k=1}^{n} \sigma_{\theta_{k}}^{2}\left|\Re\left(\mathcal{J}_{k}^{\lambda_{i}}\right) \Re\left(\mathcal{J}_{k}^{\lambda_{j}}\right)\right|$

It is possible to show that these approximations are second-order accurate in the variation coefficients of the random variables $\theta_{i}$. Though approximate, this method only requires the sampling of the random parameters, as the computation of the Jacobian and Hessian matrices is performed once and for all. However, this method depends on the convergence of the Taylor series and is not able to capture bifurcations.

\subsection{Collocation method}

The collocation is a non-intrusive interpolation method. It consists in using deterministic solvers to obtain a set of solutions and then in applying sampling methods and interpolation procedures to generate samples of the solution. Le Maître and Knio (2010) identify three main non-intrusive methods: the non-intrusive spectral projection, the least-square fitting and the collocation methods. In this work, a specific attention is devoted to the collocation method.

Let consider a subset $\Theta_{m} \subset \Theta$ containing $m$ realizations $\tilde{\theta}^{(i)} \in \Theta_{m}$ with $i=1, \ldots m$. For each realization, the eigenproblem is solved with a deterministic solver. A collection of $m$ sets of $n$ eigenvalues is constituted, whose elements $\tilde{\lambda}_{j}\left(\tilde{\theta}^{(i)}\right)$ satisfy $\mathcal{P}\left(\tilde{\lambda}_{j}\left(\tilde{\theta}^{(i)}\right)\right)=0$ with $j=1, \ldots n$. Eigenvalues are thus known for some realizations. The random space is next completed by interpolating these $m$ realizations. An approximating space $\tilde{\Theta}_{m}$ is thus constructed, with the subscript here recalling the dependence on the number of collocation points. The approximation of the eigenvalues $\boldsymbol{\Lambda}(\theta)$ on $\tilde{\Theta}_{m}$ is given by

$\tilde{\boldsymbol{\Lambda}}(\theta)=\sum_{i=1}^{m} \tilde{\boldsymbol{\Lambda}}^{(i)} \phi_{i}(\theta)$

where $\phi_{i}(\theta)$ are interpolation functions defined on the random space. Since $\tilde{\Lambda}^{(i)}$ contains exact solutions, the function $\phi_{i}(\theta)$ related to the collocation $\tilde{\theta}^{(i)}$ is equal to one when $\theta=\tilde{\theta}^{(i)}$ and zero at other collocation points. Actually, Lagrange polynomials are the least degree polynomials satisfying this constraint (Le Maître and Knio, 2010; Quarteroni et al., 2007).

The generic form of a one-dimensional Lagrange polynomial is given in Bender and Orszag (1999)

$\mathcal{L}_{\chi^{(i)}}^{m}(x)=\prod_{j=1, j \neq i}^{m} \frac{x-x^{(j)}}{\chi^{(i)}-x^{(j)}}$

with the $m$ collocation points as $x^{(i)} \in \mathbb{C}$. For each constraint $x^{(i)}$, a Lagrange polynomial interpolation is built up. For collocation in 
higher dimensions, $\mathbf{x}^{(i)} \in \mathbb{C}^{n}$, the $n$-dimensional Lagrange polynomial reads

$\mathcal{L}_{\mathbf{x}^{(i)}}^{\mathbf{m}}\left(x_{1}, \ldots x_{n}\right)=\prod_{k=1}^{n} \mathcal{L}_{x_{k}^{(i)}}^{m_{k}}\left(x_{k}\right)$,

where $m_{k}$ is the $k$ th component of $\mathbf{m} \in \mathbb{N}^{n}$ gathering the number of collocation points per dimension. A $n$-dimensional Lagrange polynomial is the product of $n$ one-dimensional Lagrange polynomials. Actually, if a similar sampling is realized in each dimension of the random space, a single group of one-dimensional Lagrange polynomials can be built without distinction on the dimension.

Applying this concept to the interpolation of an eigenvalue of $\tilde{\lambda}_{j}$ in (16), we have the expression

$\tilde{\lambda}_{j}\left(\theta_{1}, \ldots \theta_{n}\right)=\sum_{i=1}^{m} \tilde{\lambda}_{j}^{(i)} \mathcal{L}_{\tilde{\theta}^{(i)}}^{\mathbf{m}}\left(\theta_{1}, \ldots \theta_{n}\right)$.

\subsection{Galerkin approach}

The Galerkin approach (Ghanem and Ghosh, 2007) is an intrusive method, based on the spectral expansion of the random quantities involved in the problem, such as the Polynomial Chaos Expansion (PCE). For instance, the PCE of $\mathbf{B}(\theta)$ reads

$\mathbf{B}(\theta)=\sum_{i=0}^{L-1} \psi_{i}(\boldsymbol{\xi}) \mathbf{B}_{i}$

with $L$ the truncation order of the expansion, $\psi_{i}(\xi)$ the polynomial chaos (PC) and $\xi$ a set of independent identically distributed (iid) random variables. The PC $\psi_{i}(\xi)$ depends on the probability distribution of $\xi$; Askey-Wilson polynomials are usually chosen. The PC coefficients of $\mathbf{B}(\theta)$ are given by

$\mathbf{B}_{i}=\frac{E\left[\mathbf{B}(\theta) \psi_{i}\right]}{E\left[\psi_{i}^{2}\right]}$

with $E[$.$] denoting the expectation operator.$

The PCE of the $l$ th eigenvalue and the $l$ th eigenvector are

$\mathbf{Y}_{l}=\sum_{i=0}^{P-1} \psi_{i}(\boldsymbol{\xi}) \hat{\mathbf{Y}}_{l, i}, \quad \lambda_{l}=\sum_{i=0}^{P-1} \psi_{i}(\boldsymbol{\xi}) \hat{\lambda}_{l, i}$

with $P$ the number of terms in these PCE's $(L<P), \hat{\mathbf{Y}}_{l, i}$ and $\hat{\lambda}_{l, i}$ the PC coefficients of the $l$ th eigenvector and the $l$ th eigenvalue, respectively. The expansions (20) and (22) are then substituted in (12). This result is multiplied by the PC $\psi_{k}$, before computing the expectation of both members,

$\sum_{i=0}^{P-1} \sum_{j=0}^{L-1} C_{i j k} \mathbf{B}_{j} \hat{\mathbf{Y}}_{l, i}=\sum_{i, j=0}^{P-1} C_{i j k} \hat{\lambda}_{l, j} \hat{\mathbf{Y}}_{l, i}$

with $k=0, \ldots P-1$ and $C_{i j k}=E\left[\psi_{i} \psi_{j} \psi_{k}\right]$. Being probably complexvalued, the considered eigenvector and eigenvalue are split into their real and imaginary parts,

$\hat{\mathbf{Y}}_{l, i}=\hat{\mathbf{Y}}_{l, i}^{\Re}+i \hat{\mathbf{Y}}_{l, i}^{\mathfrak{\Im}}, \quad \hat{\lambda}_{i}=\hat{\lambda}_{l, i}^{\Re}+\imath \hat{\lambda}_{l, i}^{\mathfrak{T}}$

with $l=\sqrt{-1}$. The eigenproblem (23) is solved, provided the real and the imaginary parts are both equal to zero,

$$
\begin{aligned}
& \sum_{i, j=0}^{P-1} C_{i j k}\left[\mathbf{B}_{j} \hat{\mathbf{Y}}_{l, i}^{\Re}-\hat{\lambda}_{l, j}^{\Re} \hat{\mathbf{Y}}_{l, i}^{\Re}+\hat{\lambda}_{l, j}^{\Im} \hat{\mathbf{Y}}_{l, i}^{\Im}\right]=\mathbf{0} \\
& \sum_{i, j=0}^{P-1} C_{i j k}\left[\mathbf{B}_{j} \hat{\mathbf{Y}}_{l, i}^{\Im}-\hat{\lambda}_{l, j}^{\Im} \hat{\mathbf{Y}}_{l, i}^{\Re}-\hat{\lambda}_{l, j}^{\Re} \hat{\mathbf{Y}}_{l, i}^{\Im}\right]=\mathbf{0}
\end{aligned}
$$

with $\mathbf{B}_{j>L}=\mathbf{0}$ and where the superscripts $\Re$ and $\mathfrak{I}$ denote the real and the imaginary parts of the argument, respectively. As we count $2 P(n+1)$ unknowns in (25), this set of equations is "closed" by invoking the normalization condition of the eigenvectors, $\left\langle\mathbf{Y}_{l}, \mathbf{Y}_{l}\right\rangle=1$.
The same recipes, used to obtain (23), lead to

$\sum_{i, j=0}^{P-1} C_{i j k}\left\langle\hat{\mathbf{Y}}_{l, i}, \hat{\mathbf{Y}}_{l, j}\right\rangle=E\left[\psi_{k}\right]=\delta_{k 0}$

with $\delta_{i j}$ the Kronecker operator. Splitting once again real and imaginary parts, the $2 P$ complementary equations are

$$
\begin{aligned}
& \sum_{i, j=0}^{P-1} C_{i j k}\left(\left\langle\hat{\mathbf{Y}}_{l, j}^{\Re} \hat{\mathbf{Y}}_{l, i}^{\Re}\right\rangle+\left\langle\hat{\mathbf{Y}}_{l, i}^{\Im} \hat{\mathbf{Y}}_{l, i}^{\Im}\right\rangle\right)=\delta_{k 0} \\
& \sum_{i, j=0}^{P-1} C_{i j k}\left(\left\langle\hat{\mathbf{Y}}_{l, j}^{\Re} \hat{\mathbf{Y}}_{l, i}^{\mathfrak{J}}\right\rangle-\left\langle\hat{\mathbf{Y}}_{l, i}^{\mathfrak{I}} \hat{\mathbf{Y}}_{l, i}^{\Re}\right\rangle\right)=0,
\end{aligned}
$$

closing thus the set with $2 P(n+1)$ equations.

Contrary to the collocation approach, the Galerkin approach transforms the original eigenproblem into a set of nonlinear algebraic equations, losing therefore connection with efficient algorithms used to compute eigenvalues.

It must be noticed that the developments hereinabove differ from Adhikari and Friswell (2007), since complex-valued eigenvalues are considered. Moreover, this approach requires to solve a set of $2 P(n$ +1 ) equations for each eigenpair and to consider in this set the coupling between the eigenvalues and the eigenvector components, which may become computationally cumbersome.

\subsection{Flutter probability}

The flutter probability can be derived by calculating the probability that the real part of at least one of the eigenvalues is positive. The computation of (13) is the final objective of this work. Numerical integration is usually necessary, since the number of random parameters requires high-dimensional integration. The probability of instability is more conveniently computed from the probability of the system stability.

The probability (13) can be conveniently computed by Monte Carlo method, as it readily consists in evaluating the stability of eigenvalue realizations calculated with one of the previous techniques, i.e.

$\operatorname{Pr}($ stability $)=\frac{1}{\mathrm{M}} \sum_{i=1}^{\mathrm{M}} \square\left(\boldsymbol{\Lambda}^{\Re}\left(\theta^{(i)}\right) \in \Omega\right)$

with $\square(\mathcal{A})$ the indicator function of the set $\mathcal{A}, \Omega=\bigcup_{i=1}^{n}\left\{\lambda_{i}^{\Re}<0\right\}$ and $\mathrm{M}$ the number of realizations.

\section{Applications}

Comparison of the three different methods, described in Section 3, is performed for a long-span bridge model of the Golden Gate Bridge in San Francisco (California, USA). The characteristics of this model are: $l=1263 \mathrm{~m}, B=27.43 \mathrm{~m}$, section inertia $I_{0}=4.410^{6} \mathrm{~kg} \mathrm{~m} / \mathrm{m}$, deck mass $m_{0}=37$ tons $/ \mathrm{m}, \hat{C}_{L}=3.248$ and $\hat{C}_{M}=-0.178$. The inertia $I_{0}$ includes the contribution of both moving deck and main cables. The natural frequencies and the related damping ratios of the deck modes, employed in this study, are gathered in Table 1. The flutter derivatives obtained experimentally (Jain et al., 1996a) are reproduced in Figs. 1,

Table 1

Modal frequencies [Hz] and modal damping ratios of the Golden Gate Bridge deck modes (Simiu and Scanlan, 1996; Jain, 1996): AS=asymmetric, S=symmetric, $\mathrm{V}=$ vertical, $\mathrm{T}=$ torsional.

\begin{tabular}{llll}
\hline Mode & Type & Frequency & $\xi$ \\
\hline 2 & AS-V & 0.0870 & 0.005 \\
7 & AS-T & 0.1916 & 0.005 \\
8 & S-T & 0.1972 & 0.005 \\
\hline
\end{tabular}



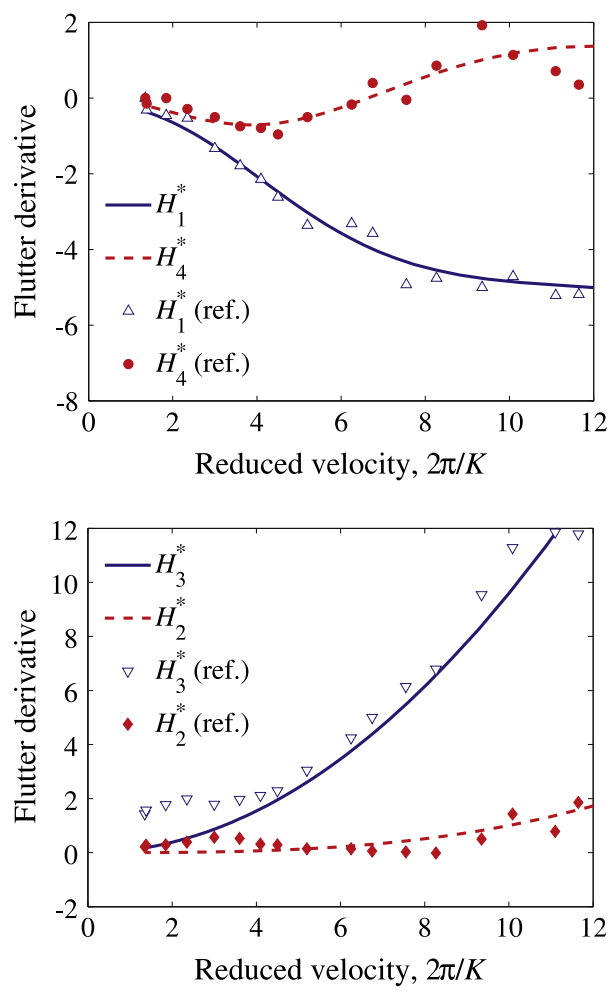

Fig. 1. Flutter derivatives $H_{1}^{*}$ to $H_{4}^{*}$ of the Golden Gate Bridge. They are considered as deterministic in the examples treated in this paper.
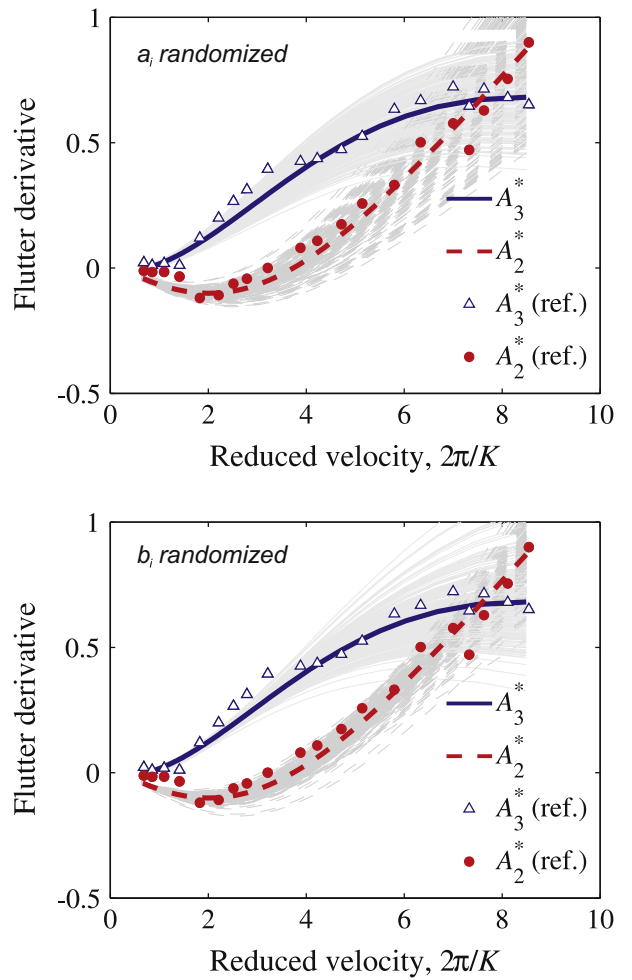

Fig. 2. Example 1: Flutter derivatives $A_{2}^{*}$ and $A_{3}^{*}$ of the Golden Gate Bridge. They are randomized with respect to the $a_{i}$ coefficients (upper plot) and to the $b_{i}$ coefficients (lower plot). In both cases, Gaussian variables are used.

2 and 4. The mean values of the corresponding indicial function coefficients $a_{i, P p}$ and $b_{i, P p}$ are gathered in Table 2. One term in the summation (5) is sufficient to properly model $\Phi_{L \alpha}$ while up to three terms are required for $\Phi_{L h}$.

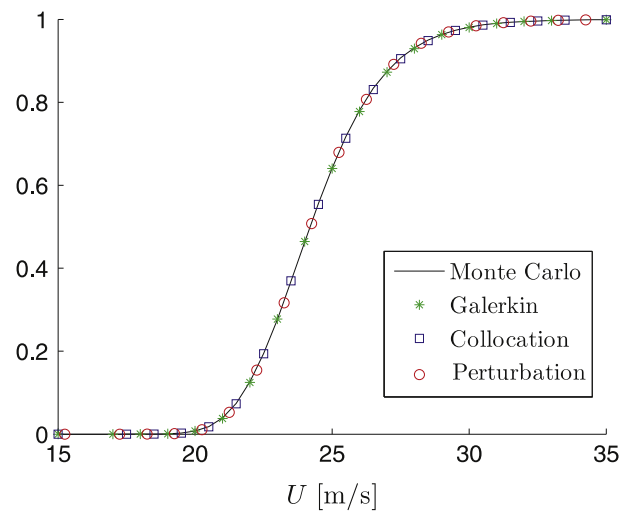

Fig. 3. Fragility curve: Probability of unstability with aerodynamic coefficients $a_{i}$ random.
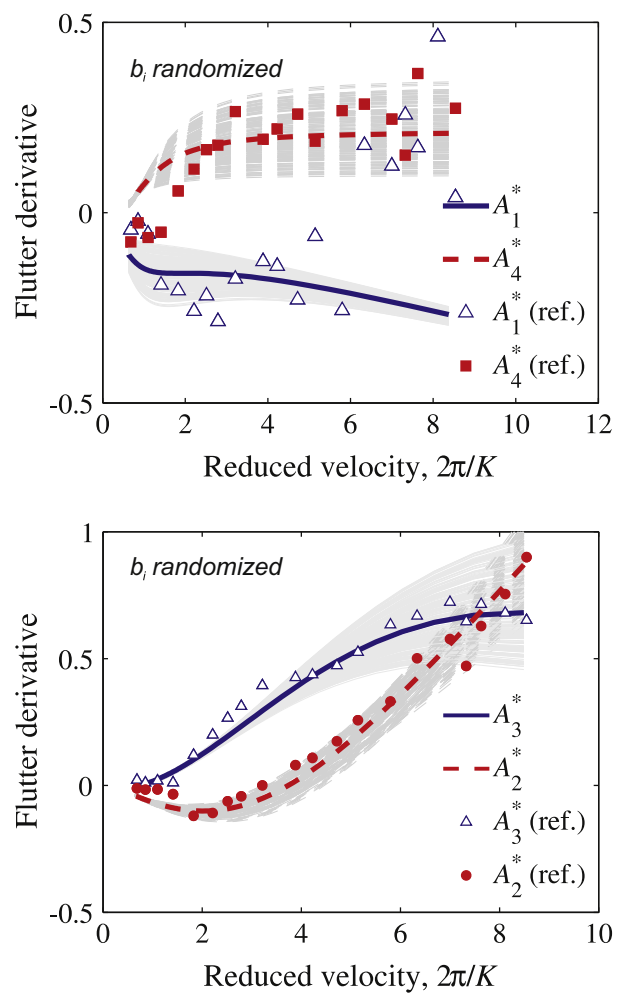

Fig. 4. Example 2: Flutter derivatives $A_{1}^{*}$ to $A_{4}^{*}$ of the Golden Gate Bridge. They are randomized with respect to the $b_{i}$ coefficients. Uniform random variables are used.

Table 2

Indicial function components $\Phi_{P p}$ for the Golden Gate Bridge.

\begin{tabular}{lrlccll}
\hline$\Phi_{P p}$ & \multicolumn{1}{c}{$a_{1, P p}$} & $b_{1, P p}$ & $a_{2, P p}$ & $b_{2, P p}$ & $a_{3, P p}$ & $b_{3, P p}$ \\
\hline$\Phi_{L h}$ & 9.71 & 0.47 & -23.87 & 0.67 & 14.767 & 0.870 \\
$\Phi_{L \alpha}$ & 2.17 & 0.036 & - & - & - & - \\
$\Phi_{M h}$ & -20.36 & 7.12 & 16.59 & 9.95 & - & - \\
$\Phi_{M \alpha}$ & -55.12 & 18.86 & 10.55 & 1.13 & - & - \\
\hline
\end{tabular}

The indicial function $\Phi_{M h}$ is described in this study by using 2 terms in the summation (5), see Table 2; additional discussion on $\Phi_{M h}$ will be provided in a later section. Following the method presented in Caracoglia and Jones (2003), the parameters shown in Table 2 are obtained by least-squares fitting of the flutter derivatives with the series of rational fractions corresponding to the general representation (5). The flutter derivatives 
reconstructed from the indicial functions with the parameters taken from Table 2 (mean values) are represented by continuous lines in Figs. 1, 2 and 4, whereas the experimental data points reproduced from Jain et al. (1996a) are depicted by discrete-point markers and listed in the legends with the designation "ref". Figs. 2 and 4 also show 200 randomized flutter derivatives for $A_{1}^{*}$ to $A_{4}^{*}$, associated with $\Phi_{M \alpha}$ and $\Phi_{M h}$; they were generated by MC sampling, with the $a_{i}$ and $b_{i}$ coefficient considered as random variables with mean values as given in Table 2 and distributions as described below. These realizations span a domain whose extent increases with increasing reduced velocity; this is aimed to model the larger measurement errors at higher velocities.

\subsection{Instability of a purely torsional mode}

First, torsional flutter is considered, as it has been suggested that aeroelastic instability can still be adequately captured for this bridge by a simplified single-mode analysis. The deck mode that is considered is the eighth purely torsional mode. The mode shape is supposed to be a half-sine function. The modal parameters are thus $I_{8}=I_{0} l / 2, Q_{8}=\rho B^{4} / I_{0}$ and $G_{\alpha_{8} \alpha_{8}}=1 / 2$.

According to Table $2\left(P_{\_} p \equiv M_{-} \alpha\right)$, two aeroelastic states must be considered. In (10), $\mathbf{B}$ and $\mathbf{X}$ are identified as

$\mathbf{B}=\left[\begin{array}{cccc}0 & 1 & 0 & 0 \\ -K_{8}^{2}+\Lambda_{8} \Phi(0) & -2 \xi_{8} K_{8} & \Lambda_{8} & \Lambda_{8} \\ a_{1} b_{1} & 0 & -b_{1} & 0 \\ a_{2} b_{2} & 0 & 0 & -b_{2}\end{array}\right]$

and $\mathbf{X}=\left[\eta_{8} \dot{\eta}_{8} \nu_{1} \nu_{2}\right]^{T}$ with $\Lambda_{8}=\hat{C}_{M} G_{\alpha_{8} \alpha_{8}} Q_{8}$.

In the sequel, the perturbation approach is limited to a first order expansion, the Galerkin approach uses a third order expansion and then six collocation points per random parameter are considered. The statistics are computed with $10^{5}$ samples.

To compare the different methods we first assume that the two coefficients $a_{i}$ are independent Gaussian random variables while coefficients $b_{i}$ are deterministic and equal to their mean values. The mean values are given in Table 2 , while the coefficients of variation are both taken equal to 0.1. As outlined earlier, Fig. 2 shows 200 realizations of the flutter derivatives generated with these random coefficients. Fig. 3 shows the fragility curves of bridge flutter instability obtained with the proposed methods and direct Monte Carlo simulation (sampling of the parameter space and resolution of (11) for each sample). Flutter fragility is defined as the probability of flutter occurrence, $F_{f l}=1-\operatorname{Pr}$ (stable), conditional on the mean value of the wind speed at deck height, $U$. In the absence of aeroelastic load error propagation, the critical flutter velocity is deterministic and constant $\left(U_{\text {cr,det }}\right)$, and the flutter fragility degenerates into the curve $F_{f l}(U)=H\left(U-U_{\text {cr,det }}\right)$ with $H$ being the Heaviside step function. In the example of Fig. 3 the deterministic flutter velocity $U_{\mathrm{cr} \text {,det }}$ is equal to approximately $22 \mathrm{~m} / \mathrm{s}$; the presence of uncertainty in $a_{i}$ leads to a non-negligible flutter occurrence below $U_{\text {cr,det }}$. Fig. 3 confirms the really good agreement between the various methods and the direct simulation approach.

Fig. 5 illustrates the PDF of the real part of the complex conjugate eigenvalues. We also notice that the distribution is nearly Gaussian. Indeed, the distribution obtained with the Taylor expansion is naturally Gaussian and it is in good agreement with the other PDFs.

The coefficients $b_{i}$ are now assumed to be two independent Gaussian variables. Fig. 2 (bottom) shows 200 realizations of the flutter derivatives generated with these random coefficients and the fragility curves are depicted in Fig. 6. As previously, the mean values are given in Table 2 and the coefficients of variation are both taken equal to 0.1 . We notice here a notable difference with the first order

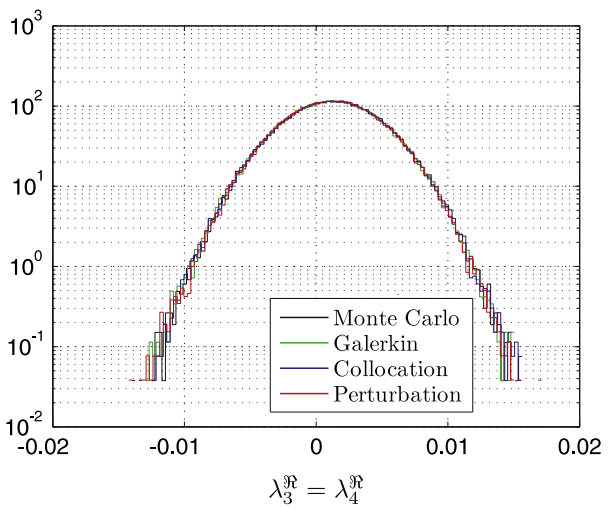

Fig. 5. PDF of the real parts of $\lambda_{3}$ and $\lambda_{4}$ (complex conjugate eigenvalues) with $a_{i}$ random and $U=25 \mathrm{~m} / \mathrm{s}$.

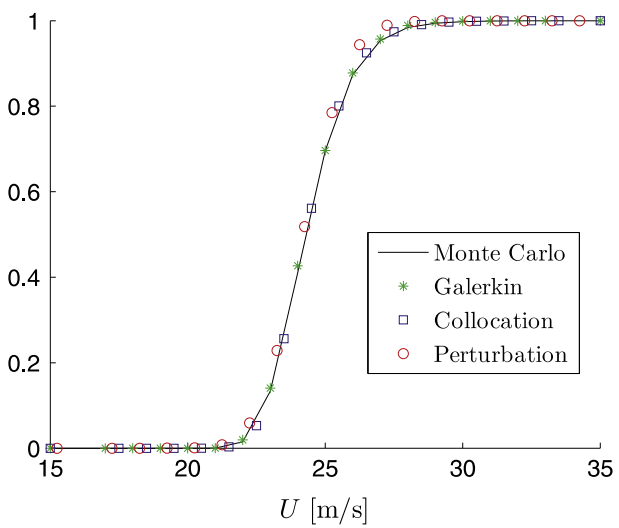

Fig. 6. Fragility curve: Probability of unstability with aerodynamic coefficients $b_{i}$ random.

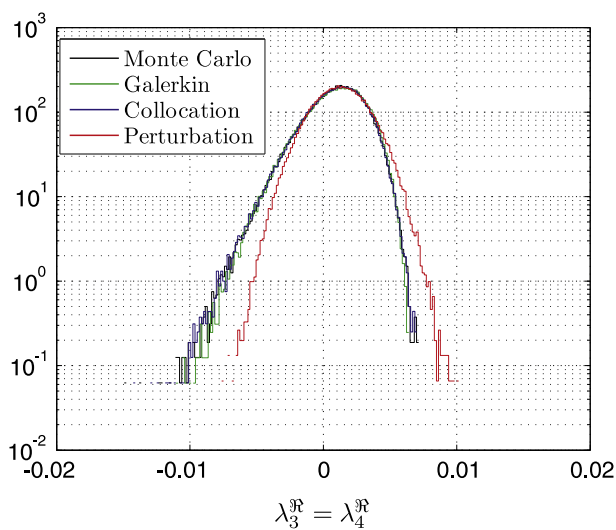

Fig. 7. PDF of the real parts of $\lambda_{3}$ and $\lambda_{4}$ (complex conjugate eigenvalues) with $b_{i}$ random and $U=25 \mathrm{~m} / \mathrm{s}$

perturbation approach to capture the randomness in the coefficients $b_{i}$, i.e., the exponents in Eq. (5).

Fig. 7 shows the PDF of the real part of the complex conjugate eigenvalues, as well. The distribution is clearly non-Gaussian, illustrating the nonlinear transformation of the random variables in the eigenproblem for the coefficients $b_{i}$. The PDF obtained by the perturbation approach, which is Gaussian, is clearly too approximate, demonstrating inadequacy of first order approximation for random $b_{i}$ coefficients.

From a computational point of view, the collocation method allows to reach accurate results in an attractively reduced computing time. This efficiency, highlighted in Figs. 3-7, is explained as the eigenproblem is only solved 36 times; also, the computation of the Lagrange 
polynomials is not cumbersome for two random parameters. In contrast, the computation using the Galerkin approach remains quite heavy even with two parameters. In particular, the necessity of separating real and imaginary parts doubles the number of equations and unknowns. Furthermore, the nonlinear set of equations must be solved for each eigenvalue at each wind velocity $U$. Therefore, the computation effort naturally increases and the calculation of the flutter fragility curves becomes an issue.

Estimation of flutter fragility is based on the hypothesis that the structural damping of all Golden Gate Bridge deck modes is equal to $0.5 \%$ with respect to critical (Table 1 ). This damping value may be considered as a plausibly safe lower bound of the structural damping. However, damping has a significant effect on the flutter probability for this bridge. This observation can be related to the fact that the Golden Gate Bridge is susceptible to single-mode torsionally driven flutter, as shown in previous work (Seo and Caracoglia, 2011) and also remarked in Jain et al. (1996b) and Jain (1996). For example, it is suggested (Seo and Caracoglia, 2011) that the generalized reliability index can almost double for this bridge if the damping ratio of the deck mode 7 (AS-T) is increased from $0.5 \%$ to $1.0 \%$ (which is however unrealistically large). The corresponding ratio between flutter probabilities is approximately equal to 20. This remark suggests that flutter fragility is extremely sensitive to structural damping. Nevertheless, a more accurate quantification of the damping effect on flutter fragility is beyond the scope of this study since the main objective is the relative comparison among several approaches for random eigenvalue analysis, applied to flutter probability estimation.

\subsection{Instability of coupled torsional and vertical modes}

In this second example, two vibration modes are considered. The coupling between the purely vertical mode $2\left(\alpha_{2}=0\right)$ and the purely torsional mode $7\left(h_{7}=0\right)$ is now included. The modal constants are $G=G_{h_{2} h_{2}}=G_{\alpha_{7} \alpha_{7}}=G_{\alpha_{7} h_{2}}=\frac{1}{2}, G_{\alpha_{2} h_{7}}=0$ and $Q_{i}=\rho B^{4} l / 2 I_{i}$ with the modal inertia $I_{2}=m_{0} B^{2} l / 2$ and $I_{7}=I_{0} l / 2$.

According to Table 2, eight aeroelastic states must be considered. For the sake of conciseness, $\mathbf{X}$ and $\mathbf{B}$ are reported in Appendix A.

To compare the different methods we only assume that the four coefficients $b_{i, M h}$ and $b_{i, M \alpha}$ are independent uniform random variables while the other coefficients are deterministic and equal to their mean values, given in Table 2 . The coefficients of variation of the random variables are taken equal to 0.1 . The corresponding randomized flutter derivatives from $A_{1}^{*}$ to $A_{4}^{*}$ are illustrated in Fig. 4 . In the sequel, the perturbation approach is limited to a first order expansion, five collocation points per random parameter are considered. The statistics are computed with $10^{4}$ samples. We do not consider the Galerkin approach in this example, as the computational time becomes extremely large in comparison with other methods, only with four parameters.

Fig. 8 shows the fragility curves of bridge flutter instability obtained with the proposed methods and direct Monte Carlo simulation. This figure confirms the good agreement between the collocation method and the direct simulation approach, while the first order Taylor expansion is too approximate. From a computational viewpoint, the collocation method allows to reach accurate results in an attractively reduced computational time. With four random variables, the computational time is reduced by 10 compared to direct Monte Carlo simulation.

Fig. 9 shows the PDF of the real part of a complex conjugate eigenvalue. As before, the nonuniform distribution illustrates the nonlinear transformation in the eigenproblem. The PDF obtained by the perturbation approach (in red) is satisfactory compared to Monte Carlo results (in blue). However, much better results are obtained with the collocation method (in black). Indeed, the PDF computed with this method and the direct Monte Carlo simulation are in really good

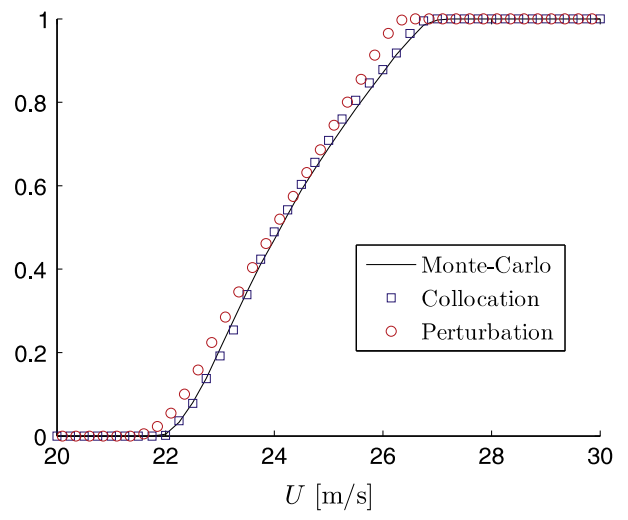

Fig. 8. Fragility curve: coupled-mode flutter probability.

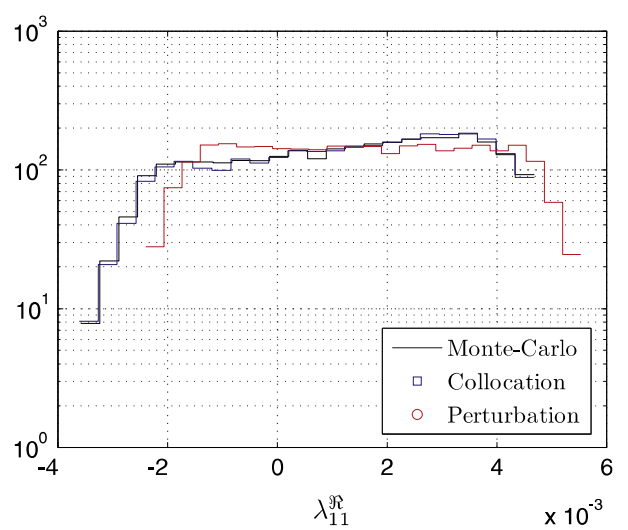

Fig. 9. PDF of the real parts of $\lambda_{11}$ (complex conjugate eigenvalue) with $b_{i, M h}$ and $b_{i, M \alpha}$ random and with $U=25 \mathrm{~m} / \mathrm{s}$. (For interpretation of the references to color in this figure caption, the reader is referred to the web version of this article.)

agreement. Both computational efficiency and accuracy of the collocation method with four random parameters are thus clearly demonstrated.

Finally, in Fig. 4 (top) the comparison between the continuous solid curve of $A_{1}^{*}$ with the experimental data with triangle marker ("ref."), suggests that the $A_{1}^{*}$ and $A_{4}^{*}$ derivatives are adequately reconstructed from the indicial function $\Phi_{M h}$ in the interval of reduced velocities between 0 and 6. Nevertheless, inadequate agreement between the $A_{1}^{*}$ data (triangle markers) and the model (continuous solid line), inherited from $\Phi_{M h}$ with 4 parameters (2 terms in Eq. (5)), is observed in Fig. 4 (top) at larger reduced velocities. A 6-parameter model (3 terms in Eq. (5)) would be needed to adequately reproduce the experimental data of $A_{1}^{*}$ and $A_{4}^{*}$ at all reduced velocities. Further investigation (not reported for brevity) has revealed that, even with an appropriately calibrated 6-parameter model for $\Phi_{M h}$ and a better representation of $A_{1}^{*}$ and $A_{4}^{*}$, the fragility curve in Fig. 8 does not significantly change. This is because stochastic bridge flutter occurs for this example in the interval of reduced velocities between 3.5 and 6.5 where the $A_{1}^{*}$ and $A_{4}^{*}$, inherited through the 4-parameter model of $\Phi_{M h}$ in Table 2, are still compatible with experimental data. In any case, the parameters of $\Phi_{M h}$ in Table 2 are not acceptable and should be replaced by the 6 -parameter model $\left(a_{1}=-8.86, b_{1}=1.73, a_{2}=12.03, b_{2}=12.67\right.$, $\left.a_{3}=3.74, b_{3}=0.304\right)$, if $A_{1}^{*}$ and $A_{4}^{*}$ of the Golden Gate Bridge were needed at other reduced velocities.

\section{Concluding remarks}

In this paper we have compared different methods for the assessment of critical flutter velocities for a bridge deck. Uncertainties resulting from the nondeterministic identification of flutter derivatives 
require the solution of an eigenvalue problem with randomly parameterized matrices. Direct Monte Carlo simulations are used as references and the problem is solved with three different probabilistic approaches. Among them, the collocation method shows a certain superiority in both accuracy and computational efficiency. This approach is thus a perfect tool to be used in higher dimensions, with more than four sources of randomness.

\section{Acknowledgements}

This work and TC's stays at Northeastern University were supported by the F.R.S.-FNRS (Fonds National de la Recherche Scientifique).

\section{Appendix A}

In example 2 , the augmented state space vector $\mathbf{X}$ collects the amplitudes and velocities in the two mode shapes as well as 8 aeroelastic states, $\mathbf{X}=\left[\eta_{2}, \dot{\eta}_{2}, \eta_{7}, \dot{\eta}_{7}, \nu_{1, L \alpha, 7}, \nu_{1, M \alpha, 7}, \nu_{2, M \alpha, 7}, \mu_{1, L h, 2}\right.$, $\mu_{2}$, Lh, $\left.2, \mu_{3, L h, 2}, \mu_{1, M h, 2}, \mu_{2, M h, 2}\right]$. The $12 \times 12$ randomly parametrized matrix $\mathbf{B}$ reads

$\mathbf{B}=\left[\begin{array}{ll}\mathbf{E} & \boldsymbol{\Lambda} \\ \mathbf{c} & \mathbf{b}\end{array}\right]$

with

$\mathbf{E}=\left[\begin{array}{cccc}0 & 0 & 1 & 0 \\ 0 & 0 & 0 & 1 \\ -K_{2}^{2} & \Lambda_{\mathrm{Lh}} \Phi_{L \alpha}(0) & -2 \xi_{2} K_{2}+\Lambda_{\mathrm{Lh}} \Phi_{\mathrm{Lh}}(0) & 0 \\ 0 & -K_{7}^{2}+\Lambda_{M \alpha} \Phi_{M \alpha}(0) & \Lambda_{M \alpha} \Phi_{\mathrm{Mh}}(0) & -2 \xi_{7} K_{7}\end{array}\right]$,

$\boldsymbol{\Lambda}=\left[\begin{array}{cccccccc}0 & 0 & 0 & 0 & 0 & 0 & 0 & 0 \\ 0 & 0 & 0 & 0 & 0 & 0 & 0 & 0 \\ \Lambda_{\mathrm{Lh}} & 0 & 0 & \Lambda_{\mathrm{Lh}} & \Lambda_{\mathrm{Lh}} & \Lambda_{\mathrm{Lh}} & 0 & 0 \\ 0 & \Lambda_{M \alpha} & \Lambda_{M \alpha} & 0 & 0 & 0 & \Lambda_{M \alpha} & \Lambda_{M \alpha}\end{array}\right]$,

$\mathbf{c}=\left[\begin{array}{cccc}0 & a_{1, L_{\alpha}} b_{1, L_{\alpha}} & 0 & 0 \\ 0 & a_{1, M_{\alpha}} b_{1, M_{\alpha}} & 0 & 0 \\ 0 & a_{2, M_{\alpha}} b_{2, M_{\alpha}} & 0 & 0 \\ 0 & 0 & a_{1, L_{h}} b_{1, L_{h}} & 0 \\ 0 & 0 & a_{2, L_{h}} b_{2, L_{h}} & 0 \\ 0 & 0 & a_{3, L_{h}} b_{3, L_{h}} & 0 \\ 0 & 0 & a_{1, M_{h}} b_{1, M_{h}} & 0 \\ 0 & 0 & a_{2, M_{h}} b_{2, M_{h}} & 0\end{array}\right]$,

and

$\mathbf{b}=\operatorname{diag}\left(-b_{1, L_{\alpha}},-b_{1, M_{\alpha}},-b_{2, M_{\alpha}},-b_{1, L_{h}},-b_{2, L_{h}},-b_{3, L_{h}},-b_{1, M_{h}},-b_{2, M_{h}}\right)$.

We have also introduced the parameters $\Lambda_{L h}=Q_{2} \hat{C}_{L} G$ and $\Lambda_{M h}=$ $Q_{7} \hat{C}_{M} G$.

\section{References}

Adhikari, S., Friswell, M.I., 2007. Random matrix eigenvalue problems in structural dynamics. Int. J. Numer. Methods Eng. 69 (3), 562-591.

Argentini, T., Pagani, A., Rocchi, D., Zasso, A., 2014. Monte Carlo analysis of total damping and flutter speed of a long span bridge: effects of structural and aerodynamic uncertainties. J. Wind Eng. Ind. Aerodyn. 128, 90-104.
Bartoli, G., Borri, C., Gusella, V., 1997. On the influence of wind turbulence on bridge deck flutter. In: Meskouris, K., Vittek, U., (Eds.), Aspects in Modern Computational Structural Analysis. Balkema, Rotterdam, Netherlands, pp. 3-17.

Bender, C.M., Orszag, S.A., 1999. Advanced Mathematical Methods for Scientists and Engineers, 2nd edition Springer Verlag, New York.

Bucher, C., Lin, Y.K., 1988. Stochastic stability of bridges considering coupled modes. J. Eng. Mech. ASCE 114 (12), 2055-2071.

Cai, C.S., Albrecht, P., 2000. Flutter derivatives based random parametric excitation aerodynamic analysis. Comput. Struct. 75 (5), 463-477.

Caracoglia, L., 2011. Simulation of linear and non-linear propagation effects of a random turbulence field on bridge flutter instability. J. Wind Eng. Ind. Aerodyn. 99 (9), 945-954.

Caracoglia, L., 2013. An Euler-Monte Carlo algorithm assessing moment Lyapunov exponents for stochastic bridge flutter predictions. Comput. Struct. 122, 65-77.

Caracoglia, L., Jones, N.P., 2003. Time domain vs. frequency domain characterization of aeroelastic forces for bridge deck sections. J. Wind Eng. Ind. Aerodyn. 91 (3), 371-402.

Cheng, J., Cai, C.S., Xiao, R.-C., Chen, S.R., 2005. Flutter reliability analysis of suspension bridges. J. Wind Eng. Ind. Aerodyn. 93 (10), 757-775. http://dx. doi.org/10.1016/j.jweia.2005.08.003.

Dragomirescu, E., Miyata, T., Yamada, H., Katsuchi, H., 2003. Probabilistic approach of structural reliability applied to bridge components. In: Proceedings of the 11th International Conference on Wind Engineering (11-ICWE), vol. 1. pp. 789-796.

Ge, Y.J., Xiang, H.F., Tanaka, H., 2000. Application of a reliability analysis model to bridge flutter under extreme winds. J. Wind Eng. Ind. Aerodyn. (2-3), 155-167.

Ghanem, R., Ghosh, D., 2007. Efficient characterization of the random eigenvalue problem in a polynomial chaos decomposition. Int. J. Numer. Methods Eng. 72 (4), 486-504.

Ghanem, R., Spanos, P., 1991. Stochastic Finite Elements. A Spectral Approach. Dover Publication, Inc., New York.

Hračov, S., Náprstek, J., Pospíšil, S., 2005. Flutter stability condition of bluff body with respect to stochastic approach. In: 10th Americas Conference on Wind Engineering (10ACWE).

Jain, A., 1996. Multi-mode Aeroelastic and Aerodynamic Analysis of Long-span Bridges (Ph.D. Dissertation), Johns Hopkins University.

Jain, A., Jones, N., Scanlan, R., 1996a. Coupled flutter and buffeting analysis of longspan bridges. J. Struct. Eng. 122 (7), 716-725.

Jain, A., Jones, N.P., Scanlan, R.H., 1996b. Coupled aeroelastic and aerodynamic response analysis of long-span bridges. J. Wind Eng. Ind. Aerodyn. 60, 69-80.

Jones, N., Scanlan, R., 2001. Theory and full-bridge modeling of wind response of cable-supported bridges. J. Bridge Eng. 6 (6), 365-375.

Kwon, S.-D., 2010. Uncertainty of bridge flutter velocity measured at wind tunne tests. In: Proceedings of the Fifth International Symposium on Computational Wind Engineering (CWE2010).

Le Maître, O.P., Knio, O.M., 2010. Spectral Methods for Uncertainty Quantification with Applications to Computational Fluid Dynamics, Scientific Computation. Springer, Netherlands.

Mannini, C., Bartoli, G., 2012. Propagazione dell'incertezza dai coefficienti aeroelastici alla velocità critica di flutter di un ponte (in italian). In: Proceedings of the Twelfth Italian Conference on Wind Engineering (IN-VENTO-2012).

Náprstek, J., Pospíšil, S., 2004. Flutter onset influenced by combined multiplicative and additive random noises. In: 5th International Colloquium on Bluff Body Aerodynamics and Applications (BBAA V). pp. 289-292.

Pospíšil, S., Náprstek, J., Hracov, S., 2006. Stability domains in flow-structure interaction and influence of random noises. J. Wind Eng. Ind. Aerodyn. 94 (11), 883-893.

Pourzeynali, S., Datta, T.K., 2002. Reliability analysis of suspension bridges against flutter. J. Sound Vib. 254 (1), 143-162.

Quarteroni, A., Sacco, R., Saleri, F., 2007. Numerical Mathematics, 2nd Edition, vol. 1 of Texts in Applied Mathematics. Springer Verlag, Berlin.

Sarkar, P.P., Tsiatas, G., 2009. Motion stability of long-span bridges under gusty winds. J. Eng. Mech. ASCE 31 (1), 158-169.

Sarkar, P.P. Caracoglia, L., Haan, F.L., Sato, H., Murakoshi, J., 2009. Comparative and sensitivity study of flutter derivatives of selected bridge deck sections. Part 1 : analysis of inter-laboratory experimental data. Eng. Struct. 31 (1), 158-169.

Scanlan, R.H., 1997. Amplitude and turbulence effects on bridge flutter derivatives. J. Struct. Eng. ASCE 123 (2), 232-236.

Scanlan, R.H., Béliveau, J.G., Budlong, S., 1974. Indicial aerodynamic functions for bridge decks. J. Eng. Mech. 100-EM4, 657-670.

Seo, D.-W., Caracoglia, L., 2011. Estimation of torsional-flutter probability in flexible bridges considering randomness in flutter derivatives. Eng. Struct. 33 (8), 2284-2296.

Seo, D.-W., Caracoglia, L., 2012. Statistical buffeting response of flexible bridges influenced by errors in aeroelastic loading estimation. J. Wind Eng. Ind. Aerodyn., 129-140. http://dx.doi.org/10.1016/j.jweia.2012.03.036.

Sepe, V., Vasta, M., 2014. Aeroelastic stability of long span bridges: comparison between quasi-deterministic and POD-based representation of the wind turbulence. In: Cunha, A., Caetano, E., Ribeiro, P., Müller, G., (Eds.), Proceedings of the 9th International Conference on Structural Dynamics, (EURODYN 2014). pp. 2855-2860. ISSN: 2311-9020, ISBN: 978-972-752-165-4.

Simiu, E., Scanlan, R., 1996. Wind Effects on Structures, 3rd edition. John Wiley and Sons, New York.

Xu, Y.-L., 2013. Wind Effects on Cable-Supported Bridges. John Wiley and Sons Singapore. 